\title{
Visible light production of hydrogen by ablated graphene: water splitting or carbon gasification?
}

\author{
Chiara Fasciani, ${ }^{a}$ Anabel E. Lanterna, ${ }^{a}$ Javier B. Giorgi* ${ }^{*}$ and Juan C. Scaiano*a \\ Department of Chemistry and Biomolecular Sciences and Centre for Catalysis Research and Innovation, University of Ottawa, 10 \\ Marie Curie, Ottawa, ON K1N 6N5, Canada.
}

Supporting Information Placeholder

\begin{abstract}
Reduced graphene oxide modified by pulsed laser ablation causes water splitting under visible light illumination $(532 \mathrm{~nm})$. When the light source is a pulsed laser, water splitting is accompanied by carbon gasification ( $\mathrm{CO}$ formation), however conventional (LED) light sources produce water splitting exclusively.
\end{abstract}

Water splitting under solar, simulated solar, or simply visible light is the subject of a rich stream of contributions to the current literature. ${ }^{1-3}$ These publications generally fall into one of two groups, those that employ a sacrificial donor and those that do not. ${ }^{4}$ Both generally produce $\mathrm{H}_{2}$ with a wide range of efficiencies, but when a sacrificial donor is used the formation of $\mathrm{O}_{2}$ is no longer possible and the reaction is not strictly considered 'water splitting.' commonly an easily oxidized molecule, such as an amine or alcohol. For example, in the case of methanol those products are $\mathrm{H}_{2}$ and $\mathrm{CH}_{2} \mathrm{O}$. In 2013, Schneider and Bahnemann have noted that "measuring $\mathrm{H}_{2}$ gas formation in such a sacrificial system no longer generates any mechanistic information". Nevertheless, to understand how the formation of hydrogen by trapping electrons can be extremely favored in the presence of sacrificial electron donors that scavenge the hole with different efficiencies, gave basic mechanism insights, helping us to design new catalytic systems.

When it comes to systems where a specific donor has not been intentionally added, the current best published result was reported by Garcia and coworkers ${ }^{6}$ using oriented gold nanoplates on graphene, as a visible light photocatalyst and yielding $1.2 \mathrm{~mol} \mathrm{H}_{2}$ per gram of composite per h (the preferred units for these reports). Many other modified carbon materials report water splitting, based on the detection of both $\mathrm{H}_{2}$ and $\mathrm{O}_{2}$, with variable degrees of success obtaining the correct $\mathrm{H}_{2} / \mathrm{O}_{2}$ stoichiometry. ${ }^{1,7}$
When carbon materials are used for water splitting, one question that is frequently not addressed is whether carbon is effectively acting as a sacrificial reducing agent. A few years ago, Matsumoto and co-workers ${ }^{8}$ suggested that graphene oxide nanosheets can generate $\mathrm{H}_{2}$ and $\mathrm{CO}_{2}$ upon photoelectrochemical conditions, using UV-light excitation and, although their electrochemical analysis was very accurate, none of the gases were characterized. A well-established reaction in the petroleum industry, referred as a carbon gasification ${ }^{9}$ and producing syn-gas (a mixture of $\mathrm{H}_{2}, \mathrm{CO}$ and some $\mathrm{CO}_{2}$ ), can be simplistically expressed as in reaction 1 and potentially competes with water splitting, reaction 2 .

$$
\begin{aligned}
& \mathrm{C}+\mathrm{H}_{2} \mathrm{O} \rightarrow \mathrm{CO}+\mathrm{H}_{2} \\
& \mathrm{H}_{2} \mathrm{O} \rightarrow 1 / 2 \mathrm{O}_{2}+\mathrm{H}_{2}
\end{aligned}
$$

Reaction 1 is endothermic and requires heat, but this is normally generated by nanomaterials under photoexcitation, where most of the radiant energy is transformed to heat, reaching high local temperatures. For example, in the case of gold nanoparticles photoexcited at $532 \mathrm{~nm}$, temperatures as high as $500^{\circ} \mathrm{C}$ have been reported. ${ }^{10}$

Although there are some recent reports studying the mechanism of graphene gasification based on metal oxidedoped graphene, ${ }^{11-12}$ it is difficult to find examples for pristine GO decomposition. Ermakov and co-workers ${ }^{13}$ have found that blue laser $(10 \mathrm{~mW}$ and $500 \mathrm{~nm}$ spot $)$ was able to burn up graphene layer-by-layer, and a nice computational study shows that the formation of $\mathrm{CO}$ would be favorable upon incomplete combustion. Garcia et al. ${ }^{14}$ have demonstrated that $\mathrm{H}_{2}$ and $\mathrm{CO}$ can be formed upon $532 \mathrm{~nm}$ laser irradiation of a GO suspension in $10 \%$ methanol aqueous solution. Although they relate the $\mathrm{CO}$ formation to structural changes on the GO, the role of the methanol in the process of $\mathrm{CO}$ formation remains unclear. However, to the best of our knowledge, no experimental detection of syn gas involving undoped carbon materials has been reported. We here out- 
line two important discoveries that would help, not only the applications in $\mathrm{H}_{2}$ but also on the $\mathrm{CO}$ production: 1) we show that visible light laser ablation of reduced graphene oxide (rGO) can generate syn gas; 2 ) the ablated $\mathrm{rGO}$ can lead to water splitting under $532 \mathrm{~nm}$ visible light illumination, following laser ablation treatment.

Two sources of rGO, namely $\mathrm{rGO}(1)$ and $\mathrm{rGO}(2)$, were used getting similar results. In a typical experiment a $\mathrm{rGO}$ aqueous suspension $(0.8 \mathrm{mg} / \mathrm{mL})$ was treated by a laser drop ablation setup using a $532 \mathrm{~nm} \mathrm{Nd-YAG} \mathrm{laser} \mathrm{(see} \mathrm{SI).}{ }^{15}$ Different numbers of shots per drop (1-20 shots for drops of 7$10 \mu \mathrm{L}$ ) were used to analyze the effect of the light on the rGO sheets. The solutions were collected in vials after irradiation and analyzed. Figure 1 shows the change in color of the samples collected after irradiation and the corresponding UV-Vis spectra. Increasing the number of shots per drop leads to a variation of the $\mathrm{rGO}$ suspension color from dark black (initial suspension) to clear grey (after 20 shots/drop); similar to changes observed in other carbon materials upon pulsed laser exposure. ${ }^{16-17}$ Note that at $532 \mathrm{~nm}$ only a small fraction of the laser pulse is absorbed by the suspension. We noticed that all the energy/power values reported refer to energy delivered, only a fraction being absorbed. Videos of the irradiated drops show the formation of gas bubbles that contain predominantly hydrogen gas (vide infra).

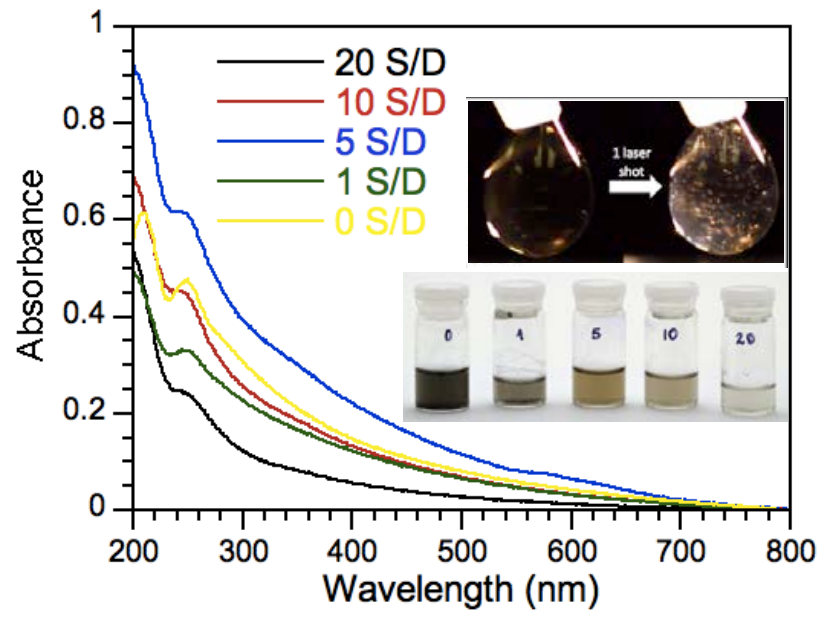

Figure 1. UV-Vis spectra of $\mathrm{rGO}(1)$ solutions after various exposures. Insets: top: drop suspension before (left) and after (right) several laser shots (the Teflon tube above the drop is 1.6 $\mathrm{mm}$ in diameter); bottom: Graphene solutions before (left) and after laser irradiation ( $532 \mathrm{~nm}$ laser, $45 \mathrm{~mJ} /$ pulse).
TEM pictures of the samples before and after ablation were collected, showing a dramatic change in morphology of the rGO flakes (Figure 2 and SI). Before laser irradiation, the electron microscopy reveals the classical graphene-like sheet conformation, no particular shape is observed, other than possible overlapping of the planes. After laser treatment, spherical shape graphene features can be detected together with the classical sheets. Increasing the number of the laser shots enhance the appearance of sphere-like morphologies. The two sources of rGO show similar behavior (Figure S3): just after one shot, the sample appears to have a spherical shape. By increasing the number of shots per drop, we observed a progressive decrease in the sizes of the spherical structures. A high surface resolution can be obtained by Second Electron imaging (SEI), which confirms the conformational change of the ablated $\mathrm{rGO}$ (1) flakes (Figure S4).

Raman spectra were recorded before irradiation and after different numbers of shots/drop rGO, showing a slight variation in the ratio between the $\mathrm{D}$ and $\mathrm{G}$ bands. ${ }^{18}$ Indeed, before ablation the intensity of the two bands are comparable (G/D 1.08), while after laser treatment the intensity of the $\mathrm{G}$ band increases, compared to the $\mathrm{D}$ band (G/D 1.131.25 ), indicating morphological changes in the structure, as seen in TEM images (see SI). As the D band is associated to defects and the presence of $\mathrm{C} \mathrm{sp}^{3}$, the laser irradiation seems to contribute to a "healing" process of the rGO, possibly related to $\mathrm{CO}$ formation from rGO defects (vide infra).

The laser ablation can be also performed by using $1 \mathrm{~mm}$ thin cuvette, where $0.3 \mathrm{~mL}$ of the rGO solution was inserted and irradiated for $30 \mathrm{~min}$, using the same $532 \mathrm{~nm}$ laser and maintaining the same laser power ( $45 \mathrm{~mJ}$ per pulse). After irradiation, the solution presented some bubbles inside the solvent and as expected the color was much clearer than the original solution. SEM pictures revealed that in this case, as in the case of the laser drop system, spherical features were obtained. For comparison, the same rGO suspension was irradiated for $2 \mathrm{~h}$ using a quad of four $532 \mathrm{~nm}$ LEDs; no change in the absorbance nor in the rGO morphology was observed by UV-Vis and SEM analysis, respectively.

The results obtained clearly indicate that the changes in rGO structure are directly correlated to the use of lasers and it does not occur if the light at the same wavelength is generated from LEDs.
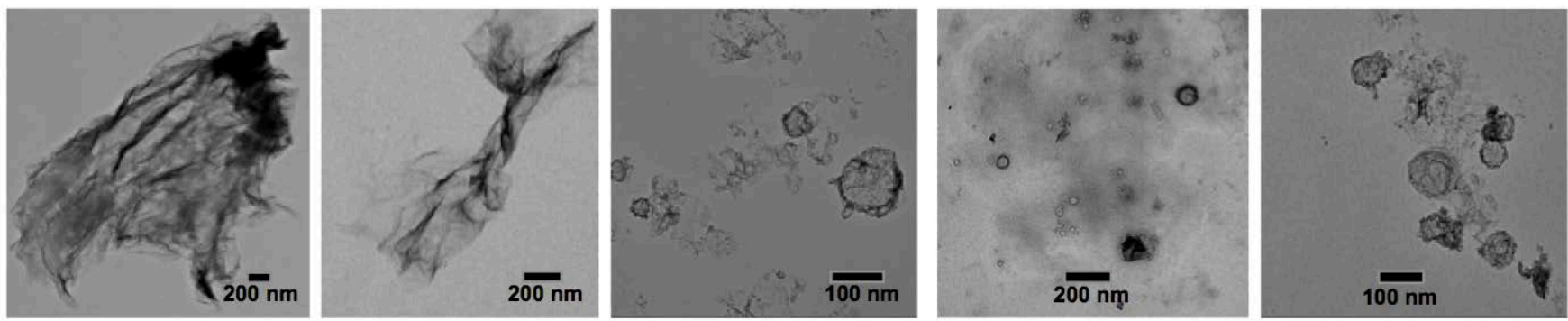
Figure 2. TEM pictures of $\mathrm{rGO}(1)$ solutions before (left) and after 1, 5, 10, 20 shots per drop (left to right).

The formation of bubbles inside the solvent can be readily observed by recording videos of the drop during the laser irradiation (inset Figure 1), or simply after irradiation of the sample in a sealed cuvette, Figure 3. In order to identify what gas was being formed, an aliquot of the reaction headspace was taken after the irradiation and injected into a gas chromatograph equipped with a packed column and a universal thermal conductivity detector (TCD), utilizing Ar as gas carrier. Analysis at the GC-TCD revealed that hydrogen gas was produced in the sample following 90 min of rGO irradiation. Thus, approximately $63 \mathrm{mmol} \mathrm{H}_{2}$ per gram of rGO are produced per $\mathrm{h}$ upon pulsed laser exposure. We note that under these conditions it is difficult to quantify the formation of $\mathrm{O}_{2}$, although a slight increase in the $\mathrm{O}_{2} / \mathrm{N}_{2}$ ratios was consistent with the production of oxygen from water splitting (eq. 2) as further discussed in the results from partial pressure experiments (vide infra).

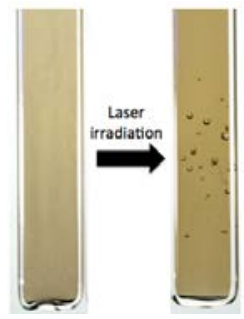

Figure 3: Picture of the graphene solution inside the $1 \mathrm{~mm}$ thick cuvette before and after $30 \mathrm{~min}$ of LED irradiation. The formation of bubbles inside the solution was observed in both LED and laser experiments.

Two potential events could be responsible for the generation of $\mathrm{H}_{2}$ : the splitting of $\mathrm{H}_{2} \mathrm{O}$ or the degradation of the graphene structure, equations 1 and 2 . In the case of reaction 2 the emission of $\mathrm{H}_{2}$ is accompanied by the production of $\mathrm{O}_{2}$. On the other hand, the evolution to form syn-gas may result from graphene degradation (reaction 1), in such a way that $\mathrm{H}_{2}$ is accompanied with the formation of $\mathrm{CO}$ or $\mathrm{CO}_{2}$. In this case, the carbon in rGO behaves as a sacrificial donor, the same way that amines or alcohols do in many reports on water splitting. Such 'assisted' water splitting does not conform to the basic chemistry and stoichiometry of reaction 2 .

Several attempts have been made to determine which of the two reactions was responsible for the $\mathrm{H}_{2}$ formation, the most successful being the use of ${ }^{18} \mathrm{O}$ isotopically labeled water. The idea behind this experiment was to monitor the release of ${ }^{18} \mathrm{O}_{2}$ or $\mathrm{CO} / \mathrm{CO}_{2}$ containing ${ }^{18} \mathrm{O}$, as in reactions 3-5.

$$
\begin{array}{r}
\mathrm{C}+\mathrm{H}_{2}{ }^{18} \mathrm{O} \rightarrow \mathrm{C}^{18} \mathrm{O}+\mathrm{H}_{2} \\
\mathrm{C}+2 \mathrm{H}_{2}{ }^{18} \mathrm{O} \rightarrow \mathrm{C}^{18} \mathrm{O}_{2}\left(\text { or } \mathrm{CO}^{18} \mathrm{O}\right)+2 \mathrm{H}_{2} \\
\mathrm{H}_{2}{ }^{18} \mathrm{O} \rightarrow{ }^{1 / 2}{ }^{18} \mathrm{O}_{2}+\mathrm{H}_{2}
\end{array}
$$

For this purpose three solutions of $\mathrm{rGO}$ in $\mathrm{H}_{2}{ }^{18} \mathrm{O}$ were prepared in a long neck cuvette and purged with Ar for 45 min. The samples were subjected to different treatments, namely rGO modification and water splitting using different light sources. The samples headspaces were then analyzed using a customized quadrupole mass spectrometer. First, a sample was irradiated with a $532 \mathrm{~nm}$ laser for $1 \mathrm{~h}$ (2 in Figure 4); second, a different sample was irradiated for $1 \mathrm{~h}$ with $532 \mathrm{~nm}$ LEDs (3 in Figure 4); and finally, the third sample was prepared and irradiated for $30 \mathrm{~min}$ with $532 \mathrm{~nm}$ laser. After the laser irradiation, the third sample was purged for another $45 \mathrm{~min}$ with Ar to make sure that any possible gas detected after the second step was not already produced during the laser irradiation. Finally, the LED irradiation was performed for $1 \mathrm{~h}$ ( 4 in Figure 4 ). Figure 4 summarizes the results found after mass analysis of the 3 samples headspace. The first sample showed the presence of ${ }^{18} \mathrm{O}_{2}$ and some labeled $\mathrm{C}^{18} \mathrm{O}$, but no labelled $\mathrm{CO}^{18} \mathrm{O}$. Particularly, $532 \mathrm{~nm}$ laser irradiation generated a mixture of gases containing 22:78 $\mathrm{C}^{18} \mathrm{O}:{ }^{18} \mathrm{O}_{2}$.

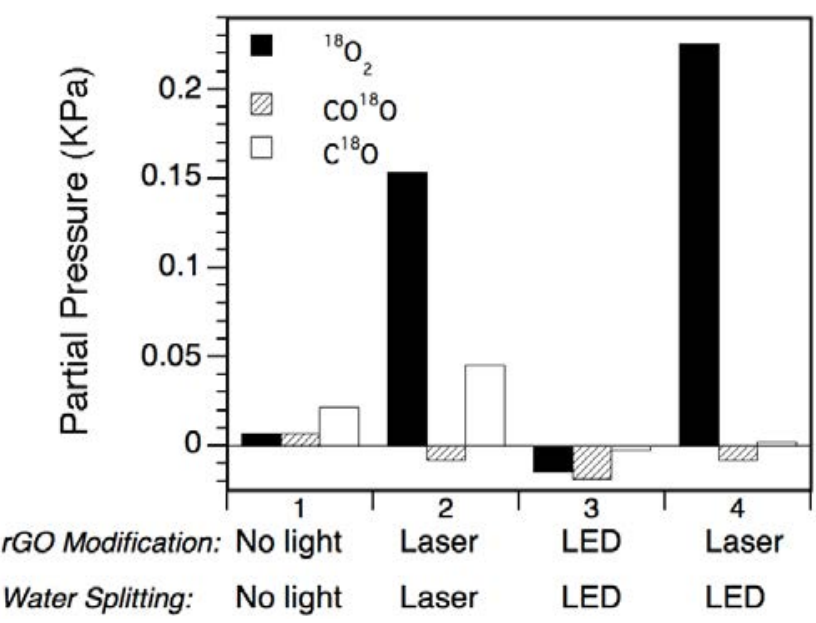

Figure 4: Comparison between ${ }^{18} \mathrm{O}_{2}$ (black), $\mathrm{C}^{18} \mathrm{O}$ (line) and $\mathrm{CO}^{18} \mathrm{O}$ (white) partial pressures of an air sample (1) and the reaction head sample of an argon purged $\mathrm{rGO}(2)$ dispersion in $\mathrm{H}_{2}{ }^{18} \mathrm{O}$ obtained after different treatments: $532 \mathrm{~nm}$ laser irradiation (2), after $532 \mathrm{~nm}$ LED irradiation (3), and $532 \mathrm{~nm}$ laser irradiation followed by $532 \mathrm{~nm}$ LED irradiation. The values are obtained comparing the areas of traces referred to each gas detected to the area of the trace relative to water vapor and multiplying the value obtained for the water partial pressure (3.2 $\mathrm{KPa})$ at $25^{\circ} \mathrm{C}$. Negative values are within experimental error of typically \pm 0.02 .

The key observation in Figure 4 is that laser irradiation leads to concurrent water splitting and syn-gas formation; thus reactions 3 and 5 occur under laser irradiation, with reaction 5 being favored; no indication for reaction 4 was observed. We note that while $\mathrm{H}_{2}$ was detected in the Laser/Laser and Laser/LED experiments in Figure 4, quantification was very challenging in these experiments, but such quantification was already achieved in the work with $\mathrm{H}_{2} \mathrm{O}$ (no ${ }^{18} \mathrm{O}$ ). While LED exposure of $\mathrm{rGO}$ did not yield any products, LED irradiation of laser pre-treated $\mathrm{rGO}(45 \mathrm{~mJ}$ 
pulses for 45 minutes as indicated above) led to significant water splitting with no syn-gas generation. This could account for some reports on $\mathrm{H}_{2}$ generation induction period; ${ }^{14}$ the restructure $\mathrm{rGO}$ is likely responsible for the $\mathrm{H}_{2}$ formation and therefore some time is needed for the restructuration of the rGO flakes before $\mathrm{H}_{2}$ is formed. Quite clearly under these conditions rGO is not a sacrificial reductant and water splitting is the only process observed. These results prove that our structural modification of the graphene sheets to spheres (see Figure 2 and SI), using laser irradiation, is promising as a method to produce catalysts for the splitting of water. This morphological modification is convenient since it occurs with visible light, in water at room temperature and can be readily controlled by the energy delivered (number of laser shots per drop).

\section{Conclusions}

There are numerous reports of solar-induced water splitting in the literature. Much has been learned from what is best described as 'assisted' water splitting, in which a sacrificial donor or reductant is employed; such systems produce hydrogen, but are not strictly water splitting, as they do not obey the chemistry or stoichiometry of reaction $2 .{ }^{4-7}$ While heavily criticized in a recent 'Commentary', it is clear that such approach was useful as a learning tool as science progressed towards true water splitting. Today's leading catalysts ${ }^{1,19}$ are in many instances carbon-based and while they have hydrogen production as a common characteristic, they usually do not test for carbon gasification (i.e., generation of $\mathrm{CO}$ or $\mathrm{CO}_{2}$ ) and those that have tested for oxygen generation have frequently faced challenges reconciling the observed $\mathrm{H}_{2} / \mathrm{O}_{2}$ stoichiometry. To the extent that our observations can be generalized, our results show that carbon gasification may be a concern under conditions of pulsed laser irradiation, but that conventional sources (visible LED light in our case) do not cause carbon gasification and that therefore rGO (and probably other graphene-like materials) do not behave as sacrificial reductants in these systems. Indeed, a reassuring observation given the growth in carbon-based water splitting photocatalysts.

\section{ASSOCIATED CONTENT}

Supporting Information. Details of methods and materials, spectroscopic data, SEM images and LED emission spectrum. The Supporting Information is available free of charge on the ACS Publications website.

\section{AUTHOR INFORMATION}

\section{Corresponding Authors}

JCS.: titoscaiano@mac.com
JBG.: Javier.Giorgi@uottawa.ca

\section{ORCID information}

JCS: 0000-0002-4838-7123

JBG: 0000-0001-9120-5594

AEL: 0000-0002-6743-0940

\section{ACKNOWLEDGMENT}

The Natural Sciences and Engineering Research Council of Canada supported this work through its Discovery programs and the award of a Vanier Scholarship to CF. Thanks are due to the Canada Research Chairs program for an award to JCS.

\section{REFERENCES}

1. Cao, S. W.; Yu, J. G., J. Photochem. Photobio. C 2016, 27, 72-99.

2. Sambur, J. B.; Chen, T. Y.; Choudhary, E.; Chen, G. Q.; Nissen, E. J.; Thomas, E. M.; Zou, N. M.; Chen, P., Nature 2016, 530 (7588), 7779.

3. Corma, A.; Garcia, H., Chem. Commun. 2004, (13), 1443-1459.

4. Jafari, T.; Moharreri, E.; Amin, A. S.; Miao, R.; Song, W. Q.; Suib, S. L., Molecules 2016, 21 (7).

5. Schneider, J.; Bahnemann, D. W., J. Phys. Chem. Lett. 2013, 4 (20), 3479-3483.

6. Mateo, D.; Esteve-Adell, I.; Albero, J.; Royo, J. F. S.; Primo, A.; Garcia, H., Nat. Commun. 2016, 7, 11819.

7. Xie, G.; Zhang, K.; Guo, B.; Liu, Q.; Fang, L.; Gong, J. R., Adv. Mater. 2013, 25 (28), 3820-3839.

8. Matsumoto, Y.; Koinuma, M.; Ida, S.; Hayami, S.; Taniguchi, T.; Hatakeyama, K.; Tateishi, H.; Watanabe, Y.; Amano, S., J. Phys. Chem. C 2011, 115 (39), 19280-19286.

9. Katta, S.; Keairns, D. L., Ind. Eng. Chem. Fund. 1981, 20 (1), 613.

10. Fasciani, C.; Alejo, C. J. B.; Grenier, M.; Netto-Ferreira, J. C.; Scaiano, J. C., Org. Lett. 2011, 13 (2), 204-207.

11. Jin, J. E.; Lee, J. H.; Choi, J. H.; Jang, H. K.; Na, J. H.; Whang, D.; Kim, D. H.; Kim, G. T., PCCP 2016, 18 (1), 101-109.

12. Ta, H. Q.; Bachmatiuk, A.; Warner, J. H.; Zhao, L.; Sun, Y. H.; Zhao, J.; Gemming, T.; Trzebicka, B.; Liu, Z. F.; Pribat, D.; Rummeli, M. H., Acs Nano 2016, 10 (6), 6323-6330.

13. Ermakov, V. A.; Alaferdov, A. V.; Vaz, A. R.; Perim, E.; Autreto, P. A. S.; Paupitz, R.; Galvao, D. S.; Moshkalev, S. A., Sci. Rep.-Uk 2015, 5, 11546.

14. Latorre-Sanchez, M.; Lavorato, C.; Puche, M.; Fornes, V.; Molinari, R.; Garcia, H., Chem Eur J 2012, 18 (52), 16774-16783.

15. Banks, J. T.; Scaiano, J. C., J. Am. Chem. Soc. 1993, 115 (14), 6409-6413.

16. Lin, T. N.; Chih, K. H.; Yuan, C. T.; Shen, J. L.; Lin, C. A. J.; Liu, W. R., Nanoscale 2015, 7 (6), 2708-2715.

17. Atienzar, P.; Primo, A.; Lavorato, C.; Molinari, R.; Garcia, H., Langmuir 2013, 29 (20), 6141-6146.

18. Alipour, R.; Jafari, A.; Elahi, A. S.; Ghoranneviss, M., Int. J. Hydrogen Energ. 2017, 42 (2), 1161-1169.

19. Mateo, D.; Esteve-Adell, I.; Albero, J.; Primo, A.; García, H., Appl. Catal. B: Environ. 2017, 201, 582-590. 
Insert Table of Contents artwork here

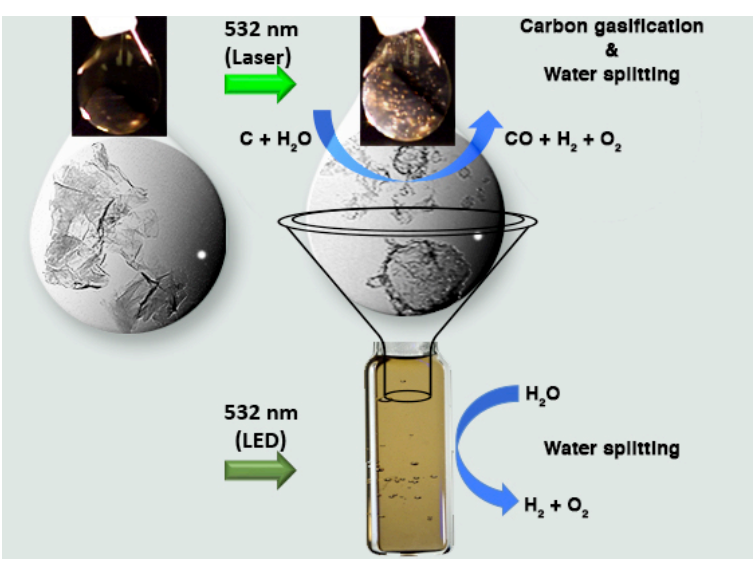

